\title{
Aspiración de proyectil de arma de fuego. Un caso poco frecuente en trauma
}

\section{Aspiration of firearm projectile. A rare case in trauma}

\author{
Juan Carlos Vázquez-Minero,* María de Jesús Villalba-Calderón, ${ }^{\ddagger}$ Carlos Daniel Vera-Márquez, \\ Derly Emmanuel Fuentes-Gómez, Teresa de Jesús Aguirre-Pérez*
}

*Instituto Nacional de Enfermedades Respiratorias Ismael Cosío Villegas, Ciudad de México, México;

†Práctica privada; ${ }^{\circledR}$ Secretaría de Salud; "Cruz Roja Mexicana Polanco.

\begin{abstract}
RESUMEN. Las heridas por arma de fuego en la actualidad son cada vez más frecuentes como consecuencia de la violencia urbana. La migración de los proyectiles a la luz de la vía aérea es muy poco frecuente y requieren de un manejo multidisciplinario. Se trata del caso de una mujer de 38 años agredida por terceras personas, con múltiples heridas por arma de fuego en rostro y cráneo que presenta proyectil inicialmente en cara, el cual posteriormente es aspirado a la vía aérea requiriendo su extracción por broncoscopia. Este tipo de pacientes requieren el manejo de varias especialidades para la resolución del problema.
\end{abstract}

Palabras clave: Cuerpo extraño, vía aérea, trauma facial, herida por arma de fuego.

\section{INTRODUCCIÓN}

La aspiración de un cuerpo extraño en adultos es muy rara, los factores de riesgos comúnmente están relacionados con la depresión de la conciencia y alteraciones del reflejo glótico. Los síntomas relacionados con la aspiración del cuerpo extraño van desde la asfixia aguda con obstrucción total o parcial, como la tos, disnea o fiebre. O bien, se pueden presentar como una atelectasia. ${ }^{1}$

Los cuerpos extraños aspirados pueden ser clasificados como orgánicos e inorgánicos. El cuerpo extraño inorgá-

\section{Correspondencia:}

\section{Dr. Juan Carlos Vázquez-Minero}

Instituto Nacional de Enfermedades Respiratorias Ismael Cosío Villegas.

Correo electrónico: minerojc@hotmail.com

Recibido: 19-IV-2021; aceptado: 07-V-2021.

Citar como: Vázquez-Minero JC, Villalba-Calderón MJ, Vera-Márquez CD, Fuentes-Gómez DE, Aguirre-Pérez TJ. Aspiración de proyectil de arma de fuego. Un caso poco frecuente en trauma. Neumol Cir Torax. 2021; 80 (3): 218-221. https://dx.doi.org/10.35366/102484

\begin{abstract}
Gunshot wounds are now becoming more frequent as a result of urban violence. Projectile migration to the lumen of the airways is very rare and requires multidisciplinary management. This is a case of a 38-year-old woman attacked by third parties, with multiple gunshot wounds to the face and craneum that initially presents a projectile in the face, which is subsequently aspirated into the airway requiring its extraction by bronchoscopy. This type of patient requires the management of several specialties to solve the problem.
\end{abstract}

Keywords: Foreign body, airway, facial trauma, gunshot wound.

nico tiende a alojarse en los bronquios del lóbulo inferior derecho. Inicialmente hay poca reacción inflamatoria y más tarde granulaciones que causan hemoptisis. La tos reaparece si el objeto cambia de posición. Puede causar atelectasia con posterior formación de absceso pulmonar o puede condicionar un enfisema obstructivo. Los cuerpos extraños orgánicos provocan una reacción inflamatoria intensa de la mucosa bronquial. Es posible que ocasionen enfisema valvular o atelectasia y posteriormente neumonía o absceso pulmonar. ${ }^{2}$

La radiografía de tórax es un elemento útil para el diagnóstico. Los objetos opacos se visualizan en la vista anteroposterior y lateral. Los objetos transparentes se sospechan cuando se evidencia en la radiografía: atelectasia, enfisema obstructivo, desplazamiento del mediastino, consolidación pulmonar o anormalidades del diafragma. Existen métodos no broncoscópicos para la extracción del cuerpo extraño como la maniobra de Heimlich; sin embargo, la broncoscopia es la técnica de elección en el tratamiento del cuerpo extraño traqueobronquial. ${ }^{3,4}$

Esta es la presentación de la aspiración de un proyectil de arma de fuego que inicialmente se encontraba en la 
cara y que posteriormente migró a la vía área y al bronquio izquierdo, requiriendo su extracción.

\section{PRESENTACIÓN DEL CASO}

Mujer de 38 años que ingresa al Servicio de Urgencias de otro hospital por ser agredida por terceras personas, con heridas por arma de fuego en cara y cráneo. A su ingreso: tensión arterial (TA) 90/60 mmHg, frecuencia cardíaca (FC) 140 lpm, frecuencia respiratoria (FR) 25 rpm, saturación de oxígeno 98\%; a la valoración inicial se evidencia vía aérea comprometida por deterioro neurológico y hemorragia a nivel de la cavidad oral. Se observa un orificio en la región temporal y uno frontal izquierda con salida de tejido cerebral; apertura palpebral forzada, se presenta anisocoria por midriasis izquierda; en cavidad oral con abundante sangrado proveniente de orificio en paladar blando, por lo que se decide manejo avanzado de la vía aérea secundario a deterioro neurológico con escala de coma Glasgow de 8.

La radiografía de tórax no muestra alteraciones, en las radiografías de cráneo se evidencia proyectil de arma de fuego (PAF) a nivel de pómulo izquierdo en la radiografía anteroposterior y lateral de cráneo (Figura 1).

Se realiza estudio tomográfico de cráneo en el que se muestra proyectil en la región maxilar derecha, con esquirla en parénquima frontal izquierdo, por lo que se realiza esquirlectomía con craniectomía por el Servicio de Neurocirugía.

Se hace cambio del tubo orotraqueal en quirófano antes de la cirugía, sin complicaciones aparentes y pasa a Terapia Intensiva. En sus primeras 24 horas de posoperatorio se evidencia disminución de la saturación de oxígeno, con disminución del murmullo vesicular en hemitórax izquierdo, submatidez a la percusión y movimientos de amplexión disminuidos, por lo que se realiza control ra- diográfico de tórax (Figura 2) en la que se observa objeto radiopaco compatible con imagen de un proyectil de arma de fuego en bronquio izquierdo y atelectasia del pulmón ipsilateral.

Es interconsultado nuestro instituto donde se valora como cuerpo extraño alojado en bronquio principal izquierdo con atelectasia pulmonar total ipsilateral. Se programa y se realiza broncoscopia flexible con videobroncoscopio 1T-180 Olympus, donde se observa el árbol bronquial con escasas secreciones espesas, se observa bronquio derecho libre y permeable y bronquio izquierdo con proyectil que obstruye el $100 \%$ de la luz. Se hace broncoscopia flexible a través de traqueoscopio de $12 \mathrm{~mm}$ (Storz) y canastilla de extracción de cuerpo extraño flexible (Boston Scientific), con lo cual se retira proyectil de arma de fuego, se hace aspiración de secreciones espesas del bronquio izquierdo para cultivo, con mucosa con inflamación sin datos de lesión de la pared bronquial y mucosa íntegra. Se egresa con saturación del 97\% (Figura 3).

Se traslada a su institución de envío donde se toma radiografía de control con adecuada expansión pulmonar bilateral (Figura 4).

Es extubada al décimo día sin complicaciones y egresada una semana después.

\section{DISCUSIÓN}

Las heridas por arma de fuego en cráneo son devastadoras, por sus consecuencias funcionales o por la alta mortalidad; el que un proyectil pase de la cara a la vía aérea es extraordinario.

Los antecedentes de la aspiración de cuerpos extraños se remontan a 1600, en donde se hizo su primera descripción. No es sino hasta el siglo XIX que se realiza la primera extracción de un cuerpo extraño de la vía aérea.
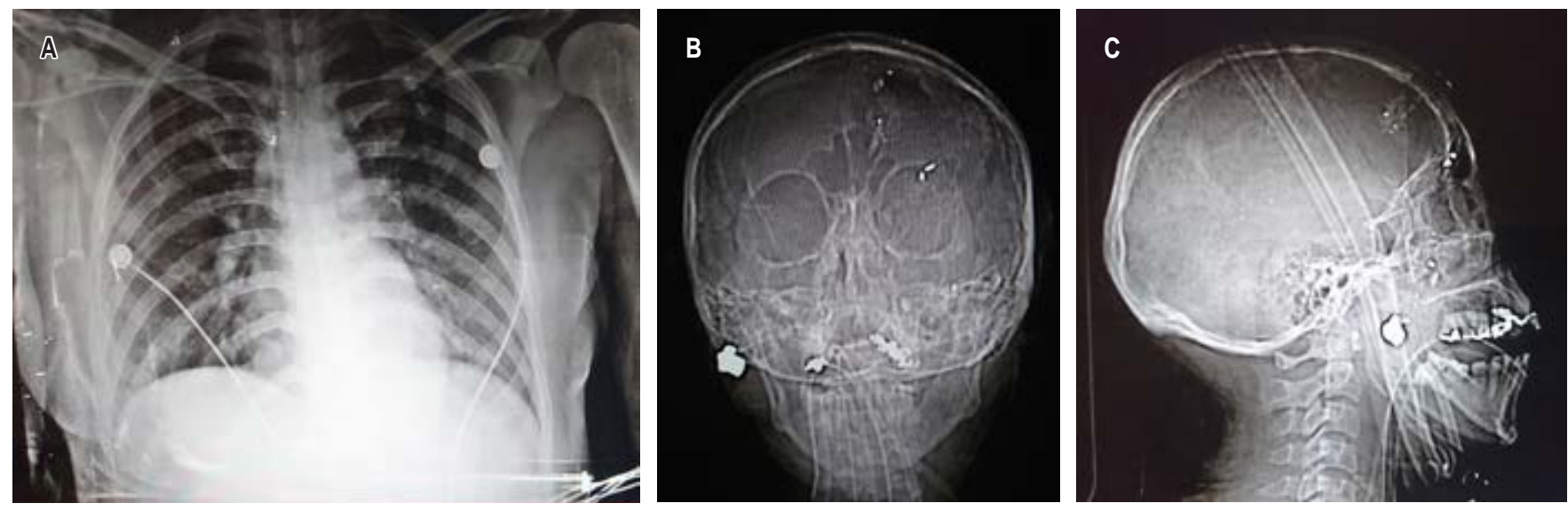

Figura 1: A) Radiografía de tórax sin alteraciones. B) Radiografía anteroposterior de cráneo con proyectil en maxilar derecho. C) Lateral de cráneo con proyectil en maxilar. 


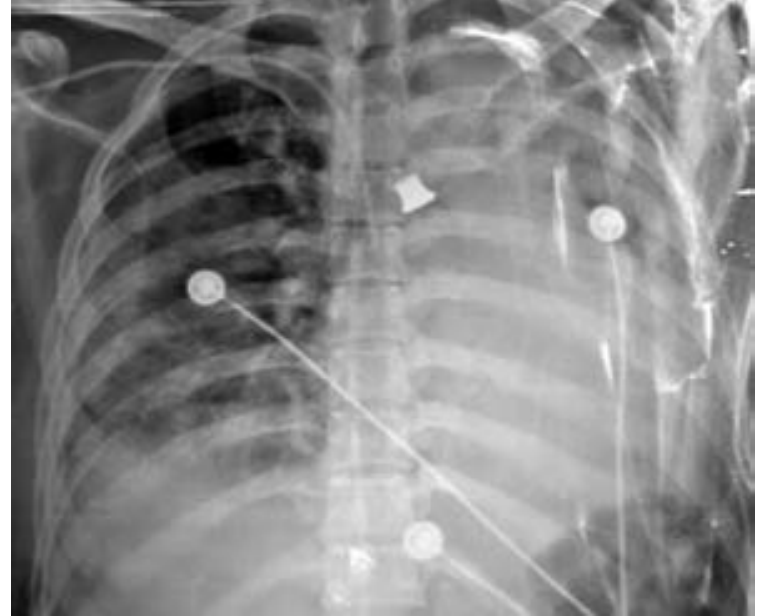

Figura 2: Radiografía de tórax con atelectasia total izquierda y proyectil en bronquio izquierdo.

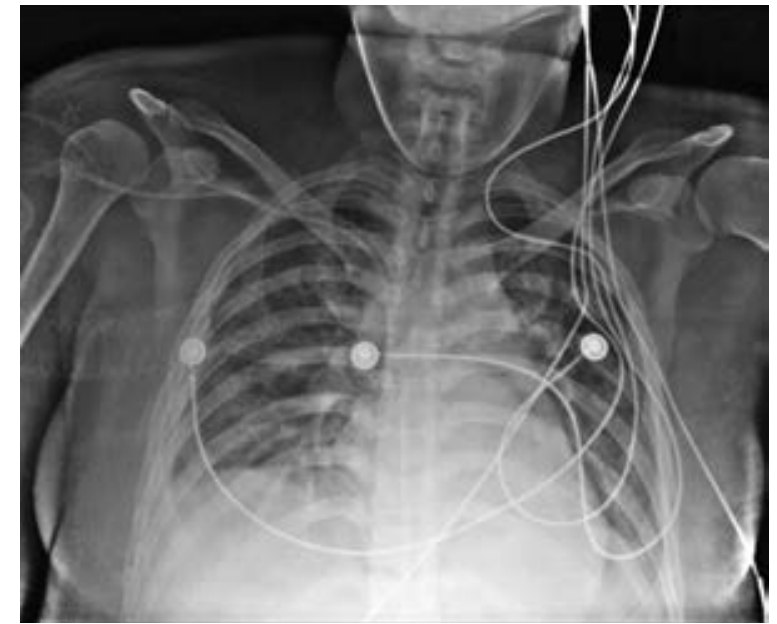

Figura 4: Radiografía de control con paciente extubado y pulmones expandidos.
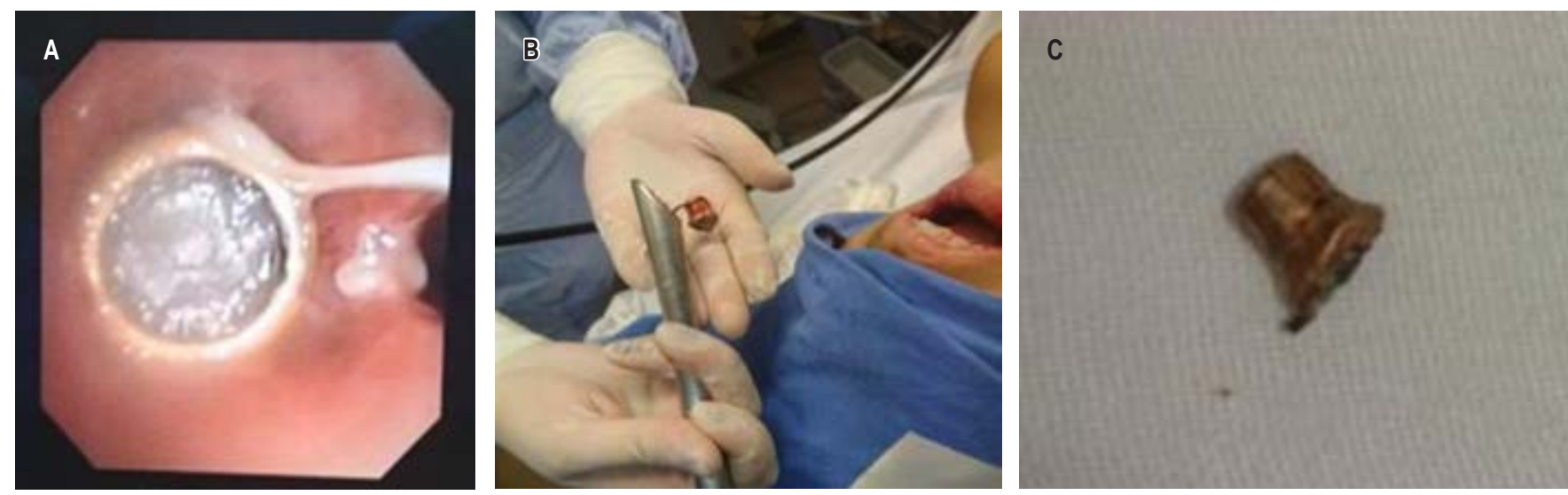

Figura 3: A) Proyectil en bronquio. B) Proyectil en canastilla y traqueoscopio. C) Proyectil extraído.

Fue Gustav Killian quien extrajo del bronquio derecho un hueso de cerdo. ${ }^{5}$

La aspiración de un cuerpo extraño de la tráquea o bronquios es más frecuente en la población pediátrica al llevarse dichos objetos a la boca durante los juegos; en el caso de los adultos no es frecuente, se asocian con alteraciones del estado mental por alcohol o drogas. En el caso de nuestra paciente, presentó alteración del estado mental por el trauma cerebral por la lesión por arma de fuego, que ameritó manejo avanzado de la vía aérea en el área de urgencias. El proyectil inicialmente se encontraba alojado en la cara con orificio de entrada en la boca. A las 24 horas presenta migración a la vía aérea, probablemente asociada a cambio de tubo endotraqueal previo a la cirugía, lo que condicionó aspiración inadvertida del cuerpo extraño a la tráquea con alojamiento en bronquio del lado izquierdo, esto como se menciona en la literatura que se asocia a manipulación de la cavidad oral o bien a procedimientos en la vía aérea. ${ }^{6}$
La clínica que los pacientes sin alteraciones en su estado mental presentan por aspiración de objetos a la vía aérea son tos y dificultad respiratoria, si este primer período es superado el paciente puede presentar estridor o sibilancias en donde se aloje el objeto. Si el cuadro avanza se condiciona atelectasia que evolucionan a infecciones de repetición o neumonías, hasta que no se sospecha o se evidencia el cuerpo extraño. En este caso por la sedación de la paciente no hubo cuadro de aspiración con tos o dificultad para respirar. Se presentaron datos de desaturación y un cuadro clínico compatible con atelectasia, se corroboró con una radiografía de tórax, que es el estudio de sospecha más importante tanto para observar la posición del cuerpo extraño como para evaluar las consecuencias del mismo como son las atelectasias o neumonías. La placa de tórax en este caso evidenció el objeto en el bronquio principal del lado izquierdo y la atelectasia total del pulmón ipsilateral.? 
La evaluación del paciente con cuerpo extraño en vía aérea parte de la sospecha diagnóstica, por datos clínicos, se complementa con estudios de imagen como es la radiografía de tórax, pero el estándar de oro para su diagnóstico preciso y su tratamiento es la broncoscopia inicialmente flexible que también en algunos casos puede ayudar a la extracción del objeto y, si se requiere, una broncoscopia rígida, que es el procedimiento recomendado para la extracción de cuerpos extraños cuando por broncoscopia flexible no es posible por el tamaño y tipo de objeto. En el caso de nuestra paciente se evaluó la vía aérea por broncoscopia flexible y después mediante broncoscopio rígido se realizó la extracción del cuerpo extraño sin ninguna complicación, con lo cual se pudo evaluar las condiciones de la tráquea y bronquios para descartar lesiones o estenosis. ${ }^{8}$

Las complicaciones que se pueden presentar en estos pacientes van desde atelectasias y neumonías hasta lesiones y estenosis del bronquio cuando pasan muchos días con el objeto, en este contexto algunas veces se requiere cirugía para su extracción. En el caso de la paciente se detectó de manera oportuna y se realizó la extracción de manera temprana. La extracción se recomienda por broncoscopia rígida, la cual requiere de entrenamiento para evitar complicaciones como lo es la lesión de la vía aérea, que en este caso no se presentó. ${ }^{9}$

\section{CONCLUSIONES}

La aspiración de cuerpos extraños en adultos es poco frecuente y el que se trate de un proyectil de arma de fuego sin lesión traqueal es extraordinario. La placa de tórax es el estudio inicial para su detección y la broncoscopia rígida es el método ideal para su extracción. Se requiere de un manejo multidisciplinario para la atención de este tipo de casos.

\section{REFERENCIAS}

1. Boyd M, Chatterjee A, Chiles C, Chin R Jr. Tracheobronchial foreign body aspiration in adults. South Med J. 2009;102(2):171-174. Available in: https://doi.org/10.1097/smj.0b013e318193c9c8

2. Horwitz S, Morgenstern H, DiPietro L, Morrison CL. Determinants of pediatric injuries. Am J Dis Child. 1988;142(6):605-611. Available in: https://doi.org/10.1001/archpedi.1988.02150060039026

3. Metha AC, Dasgupta A. Bronchoscopic approach to tracheobronchial foreign bodies in adult. Pro-flexible bronchoscopy. J Bronchol. 1997:4:173-178.

4. Ciftci AO, Bingol-Kologlu M, Senocak ME, Tanyel FC, Buyukpamukcu N. Bronchoscopy for evaluation of foreign body aspiration in children. J Pediatr Surg. 2003;38(8):1170-1176. Available in: https://doi. org/10.1016/s0022-3468(03)00263-x

5. Pérez-Fernández L. Deglución o aspiración de cuerpos extraños. Criterios Pediatr. 1991;7:89-92.

6. Benincore RA, Gutierrez MG. Aspiración de cuerpo extraño. Acta Pediatric Mex. 2019;40(3):170-179.

7. Lan RS. Non-asphyxiating tracheobronchial foreign bodies in adults. Eur Respir J. 1994;7(3):510-514. Available in: https://doi.org/10.1183 /09031936.94.07030510

8. Donando UJ, Poch ME, Casado LM. La fibrobroncoscopia en la extracción de cuerpos extraños traqueobronquiales en adultos. Arch Bronconeumol. 1998;34:76-81.

9. Guía de Práctica Clínica. Extracción de cuerpos extraños de la vía aérea en niños de 2 a 12 años en el tercer nivel de atención. México: Secretaría de Salud; 2011.

Conflicto de intereses: Los autores declaran no tener conflicto de intereses. 